\title{
M31 AND COMPANIONS: A KEY TO BAADE'S \\ STELLAR POPULATIONS THEN AND NOW
}

\author{
W. L. FREEDMAN \\ Carnegie Observatories, Pasadena, California, U.S.A. \\ (wendy@rmdm.ociw.edu)
}

\begin{abstract}
.
The stellar populations in M31 and its companions are reviewed from both historical and more modern contexts. Recent data for nearby resolved galaxies suggest that a revision of Baade's original population concept, particularly as applied to elliptical galaxies, is required. In addition, these new data highlight the difficulties in interpreting the data for, and the lack of direct information on, the more distant giant elliptical galaxies being used for cosmological studies.
\end{abstract}

\section{Introduction}

The observed properties of the bulge of M31 and its low-luminosity elliptical companions played a central role in Baade's (1944a) paper in which he outlined the concept of two distinct "population types" in galaxies. Hence, it is interesting to review the data for M31 and its companions at the marking of the 50-year anniversary of the publication of his classic paper.

Recently (Freedman, 1995), I reviewed more generally recent data on the Local Group galaxies Baade had studied (including not only the companions of M31, but also the dwarf spheroidal companion to the Galaxy). I argued that newer data on the resolved populations for many Local Group elliptical galaxies indicate considerably more recent star formation activity than did the data available to Baade; moreover, the star formation histories differ from system to system. It is my belief that if Baade were alive today, he would no longer generalize from the properties of nearby galaxies to argue for a simple picture where all (or even most) elliptical galaxies had ages comparable to the globular clusters in the Galaxy. 
In some important ways, the situation today has not changed since Baade's time: the galaxies in the Local Group remain the only galaxies whose stellar populations can be studied in detail. For nearby galaxies, the star formation rate can be measured as a function of time, and the ages, kinematics, and heavy-element abundances of stars measured directly. This situation is unlike that for more distant galaxies, where the only information that can be obtained comes from the total light (often from the nucleus alone) measured within an aperture. To date, there exists no $u$ nique method for extracting information about mean ages and abundances from integrated spectra or photometry (for example see O'Connell, 1986; Renzini and Buzzoni, 1986; Freedman, 1992b). The situation is even worse with respect to unravelling the evolutionary history of unresolved galaxy populations: it is simply not feasible with current techniques. As discussed by Freedman, either an a priori knowledge of the star formation history (SFH) or abundance distribution, or an assumption about the dispersion in either age or metallicity, is required. However, for more distant galaxies no a priori information on the SFH or abundance spread is available.

\section{Brief Historical Review: The Data available to Baade}

Using the Mount Wilson 100-inch telescope, Baade (1944a) took a series of long-exposure red plates of the bulge of M31, and its known companions NGC 221 (M32) and NGC 205. In addition, Baade (1944b) discovered and studied the stellar populations of two new low-luminosity companions, NGC 185 and NGC 147. From these plates he estimated that both the absolute magnitudes and the colors of these stars were similar to those of red giant stars observed in globular clusters in our Galaxy. Moreover, as reviewed in Freedman (1995), the discovery of RR Lyrae stars in the lowluminosity elliptical galaxies Sculptor, Draco, Leo II and Ursa Minor, and color magnitude diagrams of these objects revealing giant-branch stars with colors and luminosities resembling those in Galactic globular clusters, led Baade to conclude that these systems consisted of "pure Population II."

It is from these data on Local Group galaxies that the view emerged that in general elliptical galaxies contain stars with ages comparable to those of the Galactic globular clusters. Baade (1963) summarized his reasons for concluding that the stars in elliptical galaxies and bulges are old: "That these stars also must be old stars follows immediately ... because the integrated spectra of giant ellipticals are of types $G$ to $K$, whereas if the brightest stars were young giants we should expect type A. So it is quite clear that these stars are old stars, but of higher metal content than the halo stars."

In addition to his studies of the bulge of M31, Baade (1951) discovered 
a dense "sheet" of red ("Population II") stars throughout the disk of M31. Baade (1963) also noted a similar fainter population of red giants in the Local Group dwarf irregular galaxy IC 1613. Subsequent observations of the underlying faint red populations of stars ("Baade's red sheet") in spiral and irregular galaxies in the Local Group led to another prevalent and still current view: namely that the halos of all galaxies have ages comparable to those of Galactic globular clusters (Sandage, 1971).

\subsection{HOW WELL CAN AGES BE DETERMINED FROM OBSERVATIONS OF THE GIANT BRANCH?}

As reviewed by Blaauw (this conference), the understanding of giant branch evolution was still in a very rudimentary state at the time of the Vatican Conference in 1957. The light of elliptical galaxies at visible and redder wavelengths is dominated by that of giant branch stars. As discussed in more detail in Freedman (1995), it is now known that the giant branch luminosity is not very sensitive to age for the range 7 to 20 Gyr. The conclusion that elliptical galaxies must be old because they contain giant stars with the same luminosities and colors as those observed in Galactic globular clusters is therefore based on a faulty premise that no longer holds. Moreover, the observed colors of elliptical galaxies are very sensitive to abundance, and may be affected in some cases by the presence of dust. Hence, the interpretation of these colors as due to "old age" is problematic and the "age" estimate is not likely better than a factor of 2 or 3 of the age of the Universe; that is, not a very stringent constraint!

\section{Recent Studies: The Older Stellar Population in M31}

M31, the most luminous galaxy in the Local Group, is the closest spiral galaxy to the Milky Way. Yet it is sufficiently distant that the mainsequence turnoff luminosity for its old stellar population cannot be measured directly. The turnoff luminosity for a Galactic globular-cluster age population corresponds to $\mathrm{M}_{V} \sim 29 \mathrm{mag}$ at the distance of M31. Moreover, simple detection of such stars is not the only issue since crowding effects will be severe as this underlying population resolves. However, the availability of an Advanced Camera on the Hubble Space Telescope (HST) should provide a means of addressing questions about the $\mathrm{SFH}$ in, and formation of, the halo of M31: is there an age gradient in the halo, for example? At present, studies of the older stellar populations in M31 are limited to those of the giant branch, and now with HST, extended to studies of the horizontal branch. A brief review is given below on recent studies of the resolved stellar populations in the halo and bulge of M31. For a more general review including the younger ("Population I") stars in M31 see van den Bergh 
(1991); for a recent review of the M31 globular clusters see Huchra (1993).

With the advent of CCDs, several color-magnitude diagrams of giant branch stars in the M31 halo have been obtained in the last decade (e.g. Crotts, 1986; Mould and Kristian, 1986; Pritchet and van den Bergh, 1988; Christian and Heasley, 1991; van den Bergh and Pritchet, 1992; Davidge, 1993; Durrell et al., 1994). Collectively, these studies indicate the inherent difficulties in inferring both the mean metal abundance and its dispersion in even the nearest, most highly resolved galaxies such as M31. Estimates of the mean metallicity have ranged from -0.6 to -1.0 dex with a dispersion of $\sim 0.2$ to $>0.3$ dex. All studies have concluded that there is a dispersion in color larger than can be interpreted as due to photometry errors alone. Difficulties arise because measuring the intrinsic metallicity dispersion requires a knowledge of the total errors in the photometry, both from photon statistics and from crowding. In addition, the most metal-rich stars are both redder and fainter, and hence often below the detector limits (e.g., see Freedman, 1989; Bica et al., 1991). Most recently, Durrell et al. conclude that the halo stars are at least as metal rich (in the mean) as 47 Tuc with $<[\mathrm{Fe} / \mathrm{H}]>\sim-0.6 \mathrm{dex}$, and an intrinsic dispersion of 0.2-0.3. If confirmed, these results imply that the stars in the halo of M31 are more metal rich in the mean than the stars in the Galactic halo, and in addition, they are also more metal-rich than the M31 globular clusters.

A controversy has arisen recently over whether or not the bulge of M31 contains an intermediate age population. Near-infrared imaging by Rich and Mould (1991) and Rich et al. (1993) indicated the presence of luminous giants, possibly of intermediate age. The existence of such stars was brought into question by Davies et al. (1991). These authors argued that contamination of stars from the disk of M31 could explain the observed luminosity function. Subsequently, Depoy et al. (1993) noted that image crowding could also be responsible for the observed luminous stars. However, recent high resolution HST imaging in the inner bulge region of M31 (Rich and Mighell, 1995; Rich et al., 1995) suggests that the luminous stars are indeed real, and not artifacts of crowding, and that they belong to the bulge rather than the disk of M31.

\section{The Companions of M31}

Andromeda has 7 known companion elliptical galaxies of low luminosity. Four of these companions are dwarf elliptical galaxies (M32, NGC 205, NGC 185 and NGC 147) and three are of lower luminosity and termed dwarf spheroidal galaxies (Andromeda (And) I, II and III). The stellar populations of the M31 companions have been reviewed recently by Freedman (1992a, 1995) and Da Costa (1992). To avoid repetition, only a brief 
summary of the stellar populations of these galaxies will be given here.

Recent data for the M31 companions (and those of our own Galaxy) do not support a simple picture where these galaxies contain single-age, old, stellar populations like Galactic globular clusters. The M31 companions have not all followed the same evolutionary paths. For example, NGC 205 and NGC 185 contain not only a few blue supergiants (known to Baade, 1951), but also neutral hydrogen gas, CO gas (in the case of NGC 185), luminous asymptotic giant-branch stars, luminous carbon stars (in the case of NGC 205), and yellow supergiants, in addition to first ascent giant branch stars and RR Lyrae variables (e.g. see Freedman, 1995; and references therein). These data provide evidence for a complex and extended history of star formation beginning $\sim 10^{10}$ years ago and continuing on at a low level even to the present day. Luminous asymptotic giant branch (AGB) stars are also observed in M32 (Freedman, 1989; 1992a,b; Elston and Silva, 1992) and luminous carbon stars have been detected in And II (Aaronson et al., 1985). An intermediate age for the luminous population in M32 seems likely, but is not yet firmly established. In contrast, the dwarf spheroidal companions And I and III do not contain luminous AGB stars, apparently having ceased their star formation much earlier. In the case of NGC 147, Mould et al. (1983) concluded that less than $10 \%$ of the stellar population was of intermediate age; more recently, however, Davidge (1995) has observed the central region in this galaxy and apparently detected a luminous AGB component.

\section{Baade's Stellar Population Concept: Where Do We Go from Here?}

Despite the growing evidence that the evolutionary histories of many of the low-luminosity elliptical galaxies in the Local Group are anything but the single-aged systems that Baade envisaged, the idea that giant ellipticals are old, simple, stellar populations remains to this day (e.g. see the contribution by Renzini in this volume). Moreover, as Renzini argues, there are many compelling pieces of evidence that support this picture. For example, it is very difficult to understand the small observed dispersion in the fundamental plane (defined by central velocity dispersion, effective radius, and mean effective surface brightness) for elliptical galaxies if there have been significant differences in the evolutionary histories of these galaxies.

In contrast, Faber et al. (this volume) have concluded that elliptical galaxies have a wide range of ages. Whereas the standard picture holds that there is a sequence of metallicities for elliptical galaxies, on the basis of an $\mathrm{H} \beta$ line index, Faber et al. conclude that age, rather than metallicity, is driving that sequence. As these authors note themselves, however, there 
are a number of questions to address regarding the uniqueness of the $\mathrm{H} \beta$ line index as an age indicator. HST observations will be helpful in resolving this issue. For example, the M31 globular clusters tend to have enhanced $\mathrm{H} \beta$ line strengths (Burstein et al., 1984). However, recent HST data for the M31 globular cluster G1 (Rich et al, 1995) indicate that the G1 giant branch resembles that of the old Galactic globular cluster 47 Tuc. Further relative comparisons of other M31 globular clusters with a range in $\mathrm{H} \beta$ line strengths will prove very interesting in this regard. However, even if the $\mathrm{H} \beta$ line strength does prove to be a good age discriminant, it is still the case that it is sensitive only to the most recent burst of star formation in a galaxy. Disentangling the SFH and the abundance distribution from an integrated spectrum remains non-trivial.

The issue of the "ages" of elliptical galaxies is clearly not resolved at present. It is complicated by the fact that there is no means of directly measuring the turnoff age for old stellar populations in giant ellipticals (gEs). The nearest (normal) gEs are located in the Leo group of galaxies at a distance of about $10 \mathrm{Mpc}$. Hence, the turnoff luminosity for an old (15 Gyr) population occurs at $\mathrm{M}_{V} \sim 34.5 \mathrm{mag}$. This magnitude level is out of reach of both existing and planned facilities and instrumentation. Unfortunately, this issue is not likely to be resolved in the foreseeable future.

Baade (1944a) concluded that all elliptical galaxies and spiral bulges were old because (1) the giant branch populations in Galactic globular clusters appeared similar to those in low-luminosity ellipticals and bulges in the Local Group, and (2) he assumed that the giant branch was an indicator of age. Fifty years later, new data indicate recent, and in some cases extended, star formation in Local Group ellipticals, and current models show that the giant branch is not a very sensitive indicator of age.

So where do these new data leave us? Are the stellar populations of low-luminosity ellipticals in the Local Group at all representative of the more distant gE's? At present, this remains an open question. Perhaps the gE's have had simpler evolutionary histories than the low-luminosity ellipticals. It must, however, be kept in mind that the current prevalent view that elliptical galaxies are composed of simple, old, stellar populations was based entirely on data for Local Group galaxies. These new data certainly do not negate the importance of Baade's initial fundamental discovery, but at this 50th anniversary we do have an opportunity to take stock of what needs revising or rethinking, and to reformulate the questions we are asking. Otherwise there remains some danger that we may spend the next 50 years holding on to some of the same assumptions. At very least, these new data are telling us that we can no longer continue to make assumptions about the "age(s)" of ellipticals. Before progress can be made on this issue, an unambiguous means of determining both the age and abundance 
distributions from integrated light must be devised.

During the panel discussion at the end of the meeting, Ivan King noted that there were a number of terms presently in use in the field of stellar populations that bothered him (King, this volume). One of the problems was the use of the terms "Population I" and "Population II." He noted that it is an oversimplification to continue using Baade's terminology since populations are mixtures. His suggestion was that PopI / PopII be replaced by "age" and "metallicity" and that a population be described by whether it is young or old, and high or low metallicity. I agree entirely with Ivan that the terms population I and II are not very useful for describing mixtures of populations. Perhaps every astronomer has their own list of annoying terms, but unfortunately, in my case one of the most offending terms happens to be one that Ivan suggested, namely "age." I explained my objection in a comment to Ivan following his session, but I include it here.

I believe that the term "age" is meaningful only if a stellar population has a single age. The term "age" was relevant for a "population II" component which was assumed to have a Galactic globular-cluster (old) age, and it is part of the legacy of that thinking. Currently, however, use of the word "age" implicitly suggests a single-age population and prevents us from asking some of the fundamental questions that we need to address: for example, what has been the SFH over time? The term "age" is not the appropriate one if a spectral index being measured is sensitive only to the most recent burst of star formation in a galaxy. Nor is it appropriate to call an elliptical galaxy "old" if a small, old RR Lyrae component is found, or "young" if some luminous intermediate-age AGB stars are detected. We need to know the relative proportions (in total mass) of these populations: the SFH as a function of time or the distribution in age. These statements are simple and obvious, but the fact that we have continued to use terms that are misleading and inappropriate demonstrate both the power and simplicity of Baade's stellar population concept and the difficulty of measuring actual age/metallicity distributions. My own suggestion is that we append the word "distribution" to any discussion of stellar population characteristics (e.g., age/abundance/velocity/spatial).

This idea is not new. For example, in preparing for the written version of this review I reread a review article by Searle (1986). In it he says, "What would an idealized population description of a stellar system look like? It would involve, I think, a subdivision of the stellar system into significant subsystems, defined by their kinematic properties, or by their spatial distribution, and it would require the determination of the age distribution and the abundance distribution of each subsystem."

If we were to put aside some of the more traditional questions about "ages" of elliptical galaxies or the presence or absence of population I and 
II tracers, we might formulate a somewhat different list, such as:

- How did galaxy formation proceed? Did all galaxies start forming stars at the same epoch or at a variety of different times? How do the formation and evolution of low-luminosity ellipticals and high-luminosity ellipticals relate to/differ from/resemble each other? Once begun, how extended in time is the star formation activity in elliptical galaxies?

- If the star formation has been extended: Does star formation proceed in bursts? Or, has it fallen more continuously with time? How does it vary from galaxy to galaxy?

- What role do mergers/companions play? In what fraction of galaxies have they played a role? How does their importance evolve with time?

- How does the density of the environment affect the formation/evolution of galaxies?

I conclude that the terms "population I and II" and "age", or the assumption that most or all giant elliptical galaxies are old, are not helpful in the context of addressing these questions or resolving some of these issues. These terms were very helpful early on in the history of this subject, but now they may, in fact, be very limiting.

There are several ways to make progress in these areas and many programs are now ongoing which can help to address these questions. New observations of nearby galaxies being carried out with HST will allow the main-sequence turnoff ages to be measured directly for the most distant Galactic dwarf spheroidal companions (e.g., Leo I). Photometry of the horizontal branches in M31 and its companions can now be obtained with HST. One possible explanation for the enhanced $\mathrm{H} \beta$ features in the M31 globular clusters is the presence of blue horizontal branch stars; this possibility can now be directly tested for the first time. The detection of the turnoff for luminous intermediate-age stars in the M31 companions should be feasible, although reaching the turnoff magnitude for old (15 Gyr) populations will likely have to wait for the availability of an Advanced Camera on HST. This will also make it feasible to measure directly the dispersion in abundance of the brightest giant branch stars in elliptical galaxies in the Leo Group, and to detect the presence of luminous AGB stars, if present.

Progress in interpreting the integrated spectra of galaxies requires a means of breaking the degeneracy in age/metallicity. Resolving the stellar populations in M31 (and other Local Group galaxy) clusters with a range of metal abundances and ages will provide a direct means of testing the diagnostic capabilities of various line indices. 


\section{Conclusions}

A number of severe practical limitations remain that prevent a resolution of some of the outstanding questions in the field of stellar populations. (1) Direct measurements of age, metallicity, and kinematics are feasible only for the nearest, resolved galaxies (i.e., the Local Group). (2) There are no nearby gE's (the only type of ellipticals that can be observed to high redshifts). (3) All that is available for more distant $\mathrm{gE}$ 's are integrated light measurements. (4) From integrated photometry, separation of metallicity/age effects is not possible. (5) From integrated spectra, separation of metallicity/age effects is difficult, and not yet unambiguous.

For (low-luminosity) ellipticals in the Local Group we now know that their star formation histories have been very different, and in some cases, extended. For distant giant elliptical galaxies, we have no direct information on the epoch of formation nor on how star formation has proceeded since.

Overcoming some of these obstacles will require large telescopes in space $(>16 \mathrm{~m}$ diameter $)$ to resolve the main sequence turnoff for old (15 Gyr) populations in nearby gE's, and the identification of spectral features to break unambiguously the degeneracy between age and metallicity.

\section{References}

Aaronson, M., Gordon, G., Mould, J.R., Olszewski E. and Suntzeff, N., 1985, Ap. J.296, L7

Baade, W., 1944a, Ap. J.100, 137

Baade, W., 1944b, Ap. J.100, 147

Baade, W., 1951, Publ. Obs. Univ. of Michigan, No. 10, 7

Baade, W., 1963, Evolution of Stars and Galaxies, ed. C. Payne-Gaposchkin, MIT Press

Baade, W. and Swope, H.H., 1963, A. J.66, 300

Bica, E., Barbuy, B. and Ortolani, S., 1991, Ap. J.382, L15

Burstein, D., Faber, S.M., Gaskell, C.M. and Krumm, N., 1984, Ap. J.287, 586

Christian, C.A. and Heasley, J.N., 1991, A. J.101, 848

Crotts, A.P.S., 1986, A. J.92, 292

Da Costa, G.S., 1992, The Stellar Populations of Galaxies, eds. B. Barbuy and A. Renzini, Dordrecht: Kluwer, p.191

Davidge, T., 1993, A. J.409, 190

Davidge, T., 1995, The Local Group: Comparative and Global Properties, eds. A. Layden, J. Storm and C. Smith, ESO, in press

Davies, R.L., Frogel, J.A. and Terndrup, D.M., 1991, A. J.102, 1729

Depoy, D.L. el al., 1993, A. J.105, 2121

Durrell, P.R., Harris, W.E. and Pritchet, C.J., 1994, A. J.108, 2114

Elston, R. and Silva, D., 1992, A. J.104, 1360

Freedman, W.L., 1989, A. J.98, 1285

Freedman, W.L., 1992a, A. J.104, 1349

Freedman, W.L., 1992b, The Stellar Populations of Galaxies, eds. B. Barbuy and A. Renzini, Dordrecht: Kluwer, p.169

Freedman, W.L., 1995, The Local Group: Comparative and Global Properties, eds. A. Layden, J. Storm, and C. Smith, ESO, in press 
Huchra, J.P., 1993, The Globular Cluster-Galaxy Connection, eds. G.H. Smith and J.P. Brodie, ASP Conf. Series 48, 420

Mould, J.R. and Kristian, J., 1986, Ap. J.305, 591

Mould, J.R., Kristian, J. and Da Costa, G.S., 1983, Ap. J.270, 471

O'Connell, R.W., 1986, Spectral Evolution of Galaxies, eds. C. Chiosi and A. Renzini, Dordrecht: Reidel, p.321

Pritchet, C.J. and van den Bergh, S., 1988, Ap. J.331, 135

Renzini, A. and Buzzoni, A., 1986, Spectral Evolution of Galaxies, eds. C. Chiosi and A. Renzini, Dordrecht: Reidel), p.135

Rich, M. and Mould, J.R., 1991, A. J.101, 1286

Rich, M., Mould, J.R. and Graham, J., 1993, A. J.106, 2252

Rich, M. and Mighell, K., 1995, A. J., submitted

Rich, M., Mighell, K. and Freedman, W.L., 1995, in preparation

Sandage, A., 1971, Nuclei of Galaxies, ed. D.J.K. O'Connell, North-Holland, p.601

Searle, L., 1986, Stellar Populations, eds. C. A. Norman, A. Renzini and M.V. Tosi, Cambridge University Press, p.3

van den Bergh, S., 1991, Pub. A. S. P.103, 1053

van den Bergh, S. and Pritchet, C.J., 1992, The Stellar Populations of Galaxies, eds. B. Barbuy and A. Renzini, Kluwer: Dordrecht, p.161

MINNITI: Are these C stars in M32?

FREEDMAN: I have been searching for Carbon stars in M32 along with Mike Rich using data obtained at the Palomar 5-m plus 4-shooter. Unfortunately, bad weather and low throughput in one of the bandpasses has prevented us from success in this project to date.

COHEN: Cook, Aaronson and Illingworth found 2 Cepheids in M101 some years ago. I and a group of Palomar observers found 2 more, all based on groundbased observations.

FREEDMAN: I am aware of your work. But my results are for M100.

COHEN: The early papers by Frogel, Persson and myself ignored the issue of variables. We know that many LPV in Galactic globular clusters have $L>L_{R G B-t i p}$. Just the presence of luminous red stars with $L>L_{R G B-t i p}$ is not enough to ensure the presence of an intermediate age population.

FREEDMAN: Yes, I agree. Frogel and Elias (1988) showed very convincingly that in metal-rich Galactic globular clusters these are LPV's with luminosities greater than the first ascent red giant branch stars in the clusters. And this is indeed why I have followed up on the long-period variables in M32 that I discussed. It is important to establish what fraction of the luminous AGB stars are LPV's and what the period distribution of these LPV's is.

FERGUSON: Could you comment on the color distribution of the M32 AGB stars? Does the observed spread mean that the intermediate age population had to form at a range of metallicities?

FREEDMAN: No, not necessarily. For example, in the intermediate-age SearleWilson-Bagnuolo clusters of type V studied by Frogel, Mould and Blanco (1990), AGB stars are present with a very wide range of J-K colors.

HARRIS: I'd like to make a comment concerning the metallicity dispersion of the M31 data. The study by Durrell et al. that I mentioned yesterday resolves the intrinsic width of the giant branch quite well and shows it to be pretty near $\sigma[\mathrm{Fe} / \mathrm{H}]$ $=0.3$ or even a bit less; this is narrow enough, for example, to be matched by a simple one-zone model of chemical evolution. 